\title{
Valor de la determinación de la tiroglobulina sérica y de la gammagrafía mediante I-131 en el seguimiento del cáncer diferenciado de tiroides
}

\author{
G. RUIZ HERNÁNDEZ, C. VÁZQUEZ FORNER*, M.V. FLIQUETE PERIS* \\ Hospital Clínico Universitario. *Servicio de Medicina Nuclear. Instituto Valenciano \\ Oncológico (IVO). Valencia
}

VALUE OF THE DETECTION OF SERUM THYROGLOBULIN AND I-
131 SCINTIGRAPHY IN THE FOLLOW-UP OF DIFFERENTIATED THYROID CANCER

\section{RESUMEN}

Objetivo: El objetivo de nuestro estudio ha sido conocer la sensibilidad de la determinación de la tiroglobulina sérica y de la gammagrafía corporal mediante I-131 en la detección de tejido tiroideo residual y de la enfermedad loco-regional y a distancia; así como el valor predictivo de la tiroglobulina en el seguimiento de pacientes con cáncer diferenciado de tiroides.

Método: Para ello hemos realizado un estudio retrospectivo de 218 enfermos con cáncer diferenciado de tiroides, diagnosticados y tratados de forma homogénea, en los que se había solicitado la determinación de tiroglobulina (TG) y la gammagrafía con I-131 tras efectuar la intervención quirúrgica y entre los 6 y los nueve meses posteriores a la misma, valorando ambas pruebas de "screening" en situación hormonal de hipotiroidismo. Se han excluido del estudio 16 pacientes con anticuerpos antitiroglobulina y 14 enfermos por carecer de un seguimiento completo.

Resultados: Se ha obtenido una correlación entre ambas técnicas del $92 \%$, con una sensibilidad TG/scan para la detección de recidiva local de 85 y $90 \%$ respectivamente y superior al $90 \%$ para ambas pruebas en el diagnóstico de metástasis óseas y pulmonares. En el estudio de la afectación ganglionar regional y del tejido tiroideo residual, la sensibilidad del scan fue del $100 \%$ para la gammagrafía con I-131 e inferior al $70 \%$ para la tiroglobulina sérica. La determinación de la TG demostró un valor predictivo positivo del $99 \%$, con un valor predictivo negativo del $91 \%$ y un total de 24 resultados falsos negativos.

Conclusiones: Considerando nuestro trabajo, creemos que ambas exploraciones son complementarias, con una sensibilidad subóptima de la tiroglobulina en la detección de la afectación ganglionar loco-regional y del tejido tiroideo residual y un valor predictivo negativo que no permite realizar el seguimiento de pacientes con carcinoma diferenciado de tiroides, basándose únicamente en los resultados de este marcador tumoral.

PALABRAS CLAVE: Tiroglobulina sérica. Cáncer diferenciado de tiroides. Gammagrafía mediante I-131.

\begin{abstract}
Objectives: The objective of this work has been to know the sensiti vity of serum thyroglobulin and I-131 scintigraphy in the detection of thyroid remnants or metastases, and to evaluate the predictive value of serum thyroglobulin in the follow-up of differentiated thyroid cancer patients.

Methods: We have carried out a retrospective study of 218 patients with differentiated thyroid cancer, with detection of serum thyroglobulin levels and I-131 scintigraphy in clinical status of hypothyroidism, after to perform thyroidectomy and between six and nine months later. We have rejected 16 and 14 patients who have had presented high serum levels of thyroglobulin, or an incomplete follow-up.

Results: Our results showed a good correlation of both parameters (92\%). The sensitivity of TG and I-131 scan was $85 \%$ and $90 \%$ respecti vely, and superior to $90 \%$ for both procedures in the diagnosis of the osseous and lung metastases. The sensitivity of I-131 scan and TG was $100 \%$ and less to $70 \%$ in the detection of thyroid remnants and regional lymphatic nodes. The positive and negative value of TG was $99 \%$ and $91 \%$, respectively, with a total of 24 false negative results.

Conclusions: Considering this study, we believe that both techniques are complementary, with a lower sensitivity of serum TG in the detection of thyroid remnants and regional lymphatic involvement, and a negative predictive value that does not permit to accomplish the follow-up of dif ferentiated thyroid cancer patients, considering exclusively the results of this tumoral marker.
\end{abstract}

KEY WORDS: Serum thyroglobulin. Differentiated thyroid cancer. I131 scintigraphy.

Ruiz Hernández G, Vázquez Forner C, Fliquete Peris MV. Valor de la determinación de la tiroglobulina sérica y de la gammagrafía mediante I-131 en el seguimiento del cáncer diferenciado de tiroides. An Med Interna (Madrid) 2000; 17: 642-646.

\section{INTRODUCCIÓN}

Después de efectuar la tiroidectomía subtotal o total y el tratamiento con radioyodo, el seguimiento del cáncer de tiroi- des incluye de forma tradicional la realización de la gammagrafía de cuerpo entero mediante I-131 y la determinación de los niveles séricos de la tiroglobulina bajo el estímulo de la hormona endógena estimuladora de la glándula tiroides

Trabajo aceptado: 17 de Abril de 2000

Correspondencia: Gloria Ruiz Hernández. C/ Jesús, nº 12 - 46388 Godelleta (Valencia) 
(TSH), tras la interrupción de la terapia substitutoria con tiroxina (21). Los numerosos estudios desarrollados a lo largo de la literatura han demostrado que una elevación de los niveles de la tiroglobulina sérica, asociados o no a una gammagrafía con I-131 positiva, indican la presencia de algún resto de tejido tiroideo. Algunos trabajos han sugerido que la determinación de los niveles de la tiroglobulina sérica constituye un método con una sensibilidad como mínimo igual a la de la gammagrafía con I-131 y que en la mayoría de los casos podría incluso substituirle (2-4,7). Sin embargo, otros investigadores han encontrado que el análisis de los niveles séricos de tiroglobulina y de la captación de I-131 se basan en funciones distintas del tejido tiroideo, así como que la información que nos proporcionan ambos métodos es complementaria y que no pueden ser considerados como procedimientos alternativos en el seguimiento del cáncer diferenciado de tiroides $(11,20,24,26)$. En la asistencia diaria, podemos encontrar enfermos con niveles séricos de tiroglobulina bajos o indetectables, con gammagrafía de I-131 positiva.

El objetivo de nuestro estudio es analizar la sensibilidad de las dos técnicas de "screening" en la detección de tejido tiroideo residual y de la enfermedad regional y a distancia; así como evaluar el valor predictivo positivo y negativo de la determinación sérica de tiroglobulina en el seguimiento de pacientes con cáncer diferenciado de tiroides.

\section{MATERIAL Y MÉTODOS}

Nuestro estudio es de tipo retrospectivo, incluye a 218 enfermos con cáncer diferenciado de tiroides, de los cuales disponemos de un seguimiento completo clínico, analítico, gammagráfico y radiológico en 213 pacientes. La distribución por sexo se correspondió con un total de 169 mujeres y 35 hombres, con un intervalo de edad entre 12 y 79 años y una edad media de 47 años. Se han excluido 16 enfermos (7\%) que presentaron niveles patológicos de anticuerpos antitiroglobulina. De los 204 pacientes estudiados anatomopatológicamente, 132 fueron carcinomas papilares, 46 carcinomas foliculares y 26 carcinomas papilo-foliculares.

En todos los enfermos se efectuó tiroidectomía total o subtotal, ablación de restos tiroideos con I-131 y terapia hormonal substitutoria con tiroxina, valorando los resultados de la determinación de la tiroglobulina (TG) sérica y de la gammagrafía corporal con I-131, en situación de hipotiroidismo, entre los seis y los nueve meses de la realización del tratamiento quirúrgico. Se practicó de forma concomitante examen clínico y exploraciones diagnósticas de imagen para confirmar o excluir en los casos necesarios los hallazgos de la tiroglobulina y del scan con I-131. De los 204 enfermos valoramos en total 376 gammagrafías con I-131, con sus correspondientes determinaciones séricas de TG, con el objeto de efectuar un estudio de sensibilidad y de valor predictivo de ambas técnicas.

-Análisis de tiroglobulina sérica: de forma simultánea al rastreo corporal con I-131 se efectuó determinación de T4 total y libre, T3 y TSH, para constatar la situación clínico-analítica de hipotiroidismo, mediante radioinmunoanálisis (RIA). El análisis de la tiroglobulina sérica fue estudiado con técnica radioinmunométrica, considerando como límite superior de normalidad un valor inferior a $60 \mathrm{ng} / \mathrm{ml}$.

-Protocolo gammagráfico: el estudio gammagráfico con I-131 se realizó entre los 6 y los 9 meses posteriores a la inter- vención quirúrgica, tras la suspensión durante 4 semanas del tratamiento con tiroxina, administrando triyodotironina en las dos primeras semanas y recomendando a los pacientes una dieta pobre en iodo al menos durante la última semana anterior a la realización del scan. Tras la administración de $5 \mathrm{mCi}$ (185 MBq) de I-131, se efectuó el scan con I-131 a las 24 y 48 horas en una gammacámara Anger, equipada con un colimador de alta energía y orificios paralelos, con ventana al $20 \%$ centrada en el fotopico de $364 \mathrm{kev}$. Las imágenes fueron adquiridas con un mínimo de 20.000 cuentas o con una duración de 20 minutos, en proyecciones anteriores y posteriores que incluyeran región craneal y cervical, tórax y abdomen, practicando proyecciones opcionales si se requerían. Si la gammagrafía con I-131 demostraba una fijación anómala del radiotrazador, se administraba una dosis terapéutica de I-131, si por el contrario era negativa se continuaba el seguimiento del paciente con scan y determinación de tiroglobulina en los seis meses posteriores al primer control.

-Método estadístico: procedimiento general de cálculo de sensibilidad y valores predictivos $(10,12)$.

\section{RESULTADOS}

Al igual que otros autores hemos obtenido una buena correlación entre los resultados de ambas pruebas, con un 92\% de ocasiones en que ambas técnicas han sido positivas en demostrar la presencia o no de patología. En la tabla I se muestran los hallazgos gammagráficos en relación con la determinación sérica de tiroglobulina. Podemos observar que hemos encontrado 7 ocasiones con gammagrafía de I-131 negativa y niveles de tiroglobulina mayores de $60 \mathrm{ng} / \mathrm{ml}$; dos determinaciones se correspondían con una recidiva local tumoral demostrada por tomografía axial computarizada (CT); en 4 casos se constató posteriormente a la administración de dosis terapéuticas de I-131 la presencia de metástasis a distancia (3 pulmonares y 1 ósea) y en un paciente no se pudo confirmar la existencia de patología, continuando actualmente en seguimiento.

\section{TABLA I}

\begin{tabular}{lcc}
\multicolumn{3}{c}{ TABLA I } \\
& \multicolumn{2}{c}{ RESULTADOS TG Y SCAN } \\
\hline TG $>60 \mathrm{ng} / \mathrm{ml}$ & $\mathrm{TG}<60 \mathrm{ng} / \mathrm{ml}$ \\
\hline Scan+ & 103 & 24 \\
Scan- & 7 & 242 \\
& 110 & 266 \\
\hline
\end{tabular}

Por el contrario, detectamos 24 casos con gammagrafía con I-131 positiva y negatividad en la determinación de los niveles séricos de tiroglobulina. En 12 enfermos (50\%) se comprobó la existencia de adenopatías cervicales; en 3 casos $(12,5 \%)$ la presencia de adenopatías supraclaviculares; en 3 pacientes $(12,5 \%)$ una afectación mediastínica; en 1 caso metástasis óseas (4\%) y en 5 enfermos $(21 \%)$ tejido residual tiroideo tras la intervención quirúrgica.

En la tabla II se expone la localización de la enfermedad en los 103 casos en que ambas exploraciones fueron positivas, así como en el resto de localizaciones estudiadas. Hemos 
TABLA II

DISTRIBUCIÓN POR LOCALIZACIÓN

\begin{tabular}{lcc}
\hline Localización & Total & Scan+/TG+ \\
\hline Tejido residual & 15 & 10 \\
Adenopatías cervicales & 31 & 19 \\
Adenopatías supraclaviculares & 7 & 4 \\
Recidiva local & 20 & 15 \\
M etástasis óseas (M O) & 18 & 16 \\
M etástasis pulmonares (M P) & 29 & 26 \\
M O + M P & 13 & 13 \\
& 133 & 103 \\
\hline
\end{tabular}

obtenido una correlación del $92 \%$ entre ambas pruebas diagnósticas, con una sensibilidad TG/scan para la detección de recidiva local de $85 \%$ y $90 \%$ respectivamente. La sensibilidad de ambas técnicas para el diagnóstico de metástasis óseas fue del $97 \%$ y para las metástasis pulmonares del $93 \%$ para el scan y del $100 \%$ para la TG sérica. Llama la atención que en el diagnóstico de adenopatías locoregionales y de tejido tiroideo residual post-intervención quirúrgica, la sensibilidad del scan fue en ambos casos del $100 \%$ frente a un $60,5 \%$, y $66,6 \%$ obtenido mediante la determinación de tiroglobulina para estas dos situaciones (Tabla III).

La determinación de la TG demostró un valor predictivo positivo del $99 \%$, con un valor predictivo negativo del $91 \%$ y un total de 24 enfermos en los que la gammagrafía con I-131 fue positiva, a pesar de la normalidad de los niveles séricos de tiroglobulina.

\section{TABLA III}

\section{SENSIBILIDAD (S): TG/SCAN}

\begin{tabular}{lcc}
\hline & (S) TG & (S) Scan \\
\hline Recidiva local & $85 \%$ & $90 \%$ \\
M etástasis óseas & $97 \%$ & $97 \%$ \\
M etástasis pulmonares & $100 \%$ & $93 \%$ \\
Tejido residual tiroideo & $66,6 \%$ & $100 \%$ \\
Adenopatías loco-regionales & $60,5 \%$ & $100 \%$ \\
\hline
\end{tabular}

\section{DISCUSIÓN}

La baja sensibilidad de la tiroglobulina en la detección de tejido tiroideo normal o neoplásico no constituye un hecho habitual en el seguimiento de pacientes con cáncer diferenciado de tiroides, pero sí que ha sido descrito en la literatura (19) de la misma forma como también ha sido considerada como el marcador ideal de tejido tiroideo residual o de enfermedad metastásica (17). Esta glicoproteína de 660,000-kd actúa de prohormona para la producción de hormonas tiroideas. Su origen específico en el tejido tiroideo ha provocado una gran difusión de su utilización como marcador tumoral en el cáncer de tiroides (33). Es bien conocido que un incremento del nivel sérico de tiroglobulina en pacientes con cáncer diferenciado de tiroides es a menudo la primera evidencia de enfermedad metastásica o de recurrencia local del cáncer tiroideo, tras la tiroidectomía y la terapia ablativa con radioyodo (4). Niveles indetectables de este marcador se han encontrado sobre todo durante la terapia tiroidea hormonal o en presencia de anticuerpos antitiroideos, que pueden en dependencia del método utilizado elevar o disminuir falsamente los niveles séricos de tiroglobulina. La prevalencia estimada de los anticuepos antitiroglobulina en la población general es aproximadamente de un $10 \%$ y en pacientes con cáncer diferenciado de tiroides del $23 \%(3,15)$.

Respecto a la terapia hormonal substitutoria, varios trabajos han demostrado una mayor sensibilidad de la determinación de la tiroglobulina después de la suspensión de la administración de tiroxina, que durante el tratamiento con la misma $(20,28)$. Este hallazgo está probablemente relacionado con la presencia de pequeños focos tumorales, que responderían a la estimulación con TSH. Girelli ME y cols. $(13,14)$ señalan un $8,2 \%$ de falsos negativos de la determinación sérica de tiroglobulina durante el tratamiento con tiroxina, aunque concluyen que pese a que su valor predictivo es adecuado como marcador en el seguimiento del cáncer de tiroides, debe ser complementado con la determinación de la tiroglobulina después de la suspensión del tratamiento.

En la práctica clínica el encontrar una gammagrafía con I131 positiva con unos niveles negativos de tiroglobulina no es un hallazgo infrecuente. Algunos investigadores como Brendel y cols. (6) muestran que las metástasis menos diferenciadas de tiroides, pueden asociarse con unos niveles más bajos de tiroglobulina. Las razones que argumentan como posible explicación son: una reducción en la síntesis o en la liberación de la tiroglobulina normal, una síntesis de una tiroglobulina anormal irreconocible por los métodos de rutina de radioinmunoanálisis, o también porque existiera un aclaramiento más rápido del plasma de la tiroglobulina sérica. Pero también conviene citar que en otros casos, la pérdida de diferenciación celular se asocia con una ausencia de la captación del radiotrazador, preservándose sin embargo la síntesis de tiroglobulina sérica (11).

Otra posible explicación al fenómeno "presencia de metástasis con fijación de I-131 y ausencia de elevación de la tiroglobulina" es la descrita como "hook effect", descrito por Spencer C y cols. en 1995. Este efecto puede aparecer en presencia de una elevada concentración de antígeno, (10 a 10.000 veces el límite superior del rango del ensayo), que excede la capacidad de unión del anticuerpo en el soporte sólido. Dicho efecto no puede ocurrir utilizando métodos inmunoradiométricos, gracias a que realizan una técnica con incubación en dos fases y eliminación del suero entre las mismas (29).

Una circunstancia especial y que ocurre en ocasiones, es la presencia de rastreo con I-131 negativo, TG durante la terapia hormonal negativa y TG tras la suspensión de la misma positiva, con visualización de metástasis tras la administración de dosis terapéuticas de I-131. Esta posibilidad aparece en 5 de 61 enfermos estudiados por Ronga y cols., (24) de los que 4 tenían afectación metastásica. Ésto parece ser debido a que las células metastásicas tiroideas podrían perder la capacidad de captación de I-131 con la utilización de dosis bajas, siendo detectables cuando se efectúa la terapia con dosis elevadas. Existe una dependencia entre la incorporación del radioyodo a la célula tumoral tiroidea y la respuesta de la adenilciclasa a la estimulación de la membrana celular in vitro, de forma que una reducción de la respuesta de la adenilciclasa se correlacionaría con una disminución de la concentración de I-131 (16, 31 ). Otros autores han demostrado que la presencia del agente ácido 13-cis-retinoico en las células foliculares in vitro, mejo- 
ra el atrapamiento del radioyodo y la unión de la TSH por las células tiroideas tumorales. Este hecho podría cambiar las actitudes terapéuticas, así como el pronóstico de los pacientes con TG y scan I-131 incongruentes y de los enfermos con TG y scan I-131 congruentes pero negativos, en el seguimiento del cáncer diferenciado de tiroides (32).

Al parecer, según nos demuestran los artículos publicados en la literatura, se puede encontrar una correlación óptima entre el scan de I-131 y la determinación de tiroglobulina sérica ante la presencia de metástasis a distancia, mientras que se señala una pobre correlación ante la existencia de restos tiroideos y metástasis linfáticas cervicales y mediastínicas, que se mostrarían ávidas para captar el radioyodo y en las que a menudo la tiroglobulina sérica es indetectable. Ramanna y cols. (22) concluyen que una elevación de la tiglobulina sérica es un buen indicador de metástasis a distancia, pero que debemos ser más cautos al interpretar metástasis locales cervicales demostradas gammagráficamente utilizando sólo el criterio de la tiroglobulina, que puede ser normal. Scheneider y cols (26) presentan cuatro casos de carcinoma diferenciado de tiroides con adenopatías metastásicas cervicales y niveles normales de tiroglobulina. Botsch $\mathrm{H}$ y cols. (5) encuentran 4 pacientes con metástasis locales y 4 de 6 enfermos con metástasis distales sin elevación concomitante de la TG. Similares hallazgos también han sido señalados por Grant y cols. (15), con 4 enfermos con evidencia de enfermedad metastásica (uno de ellos con afectación pulmonar) y niveles de TG negativos. Mertens IJ y cols. (18) publican recientemente el caso de un paciente con niveles indectables de TG sérica y metástasis óseas detectadas mediante gammagrafía con I-131.

Ronga y cols. (24) obtienen una sensibilidad de la determinación de la tiroglobulina sérica durante la terapia del 83,3\%, con una sensibilidad de la gammagrafía con I-131 sólo del $76,6 \%$. Considerando ambas pruebas en conjunto se incrementa la especificidad a un $100 \%$ y la sensibilidad a un $95,7 \%$. Estos investigadores también señalan que la sensibilidad de la determinación de la tiroglobulina tras la suspensión de la terapia hormonal es del $100 \%$, pero la especificidad es muy baja $(12,9 \%)$, y que esta determinación tendría que valorarse de forma conjunta con el análisis de TG durante la administración de tiroxina y con la gammagrafía corporal mediante I-131. Más recientemente, Roelants y cols. (23) encuentran que en el seguimiento precoz del cáncer diferenciado de tiroides la determinación de la tiroglobulina sérica tras la suspensión del tratamiento con tiroxina es menos sensible que la gammagrafía con I-131 para la demostración de tejido residual tiroideo ( $87 \%$ vs $26 \%$ respectivamente), pero que posee una información predictiva del curso de la enfermedad, de forma que aquellos pacientes con gammagrafía con I-131 positiva y niveles de TG negativos se curarían más precozmente que los enfermos en los que persisten niveles de TG patológicos.

En nuestro trabajo hemos obtenido que ambas técnicas de "screening" poseen una buena correlación, con unos resultados óptimos en cuanto a sensibilidad en el diagnóstico de recidiva local y de metástasis a distancia. Sin embargo, la sensibilidad de la determinación de la tiroglobulina en cuanto a la detección de tejido tiroideo residual y de la afectación linfática locoregional, es francamente inferior a la de la gammagrafía con I-131. El valor predictivo de la tiroglobulina supera el $90 \%$, existiendo 24 enfermos en nuestro estudio con gammagrafía mediante I-131 positiva y TG negativa.

Como nota de interés, señalar que no hemos encontrado falsos positivos de la gammagrafía corporal con I-131, que aunque no son frecuentes, sí pueden aparecer en algunas circunstancias como: la contaminación por las secrecciones y exudados del cuerpo, la patogía inflamatoria, otras neoplasias no tiroideas, e incluso la hiperplasia tímica $(25,30)$. Mencionar tan sólo que en aquellas circunstancias con aumento de los niveles séricos de tiroglobulina sin traducción en el scan con I-131, serían subsidiarias de estudio con tomografía de emisión de positrones $(8,9)$.

Autores como Schlumberger y cols. (27) identifican un porcentaje de supervivencia a los 15 años de ser diagnosticadas las metástasis secundarias a carcinoma de tiroides del $33 \%$. Otros, como Aiello y cols (1), nos recuerdan que la detección precoz de la enfermedad metastásica es importante, porque precisamente en aquellos pacientes con menor potencial metastásico, son los que más se van a beneficiar de efectuar una intervención terapéutica precoz.

Creemos que la detección precoz de la diseminación locoregional y a distancia del carcinoma diferenciado de tiroides es un objetivo a lograr inicialmente, en base a los hallazgos complementarios de la determinación sérica de la tiroglobulina y de la gammagrafía con I-131 y que ninguna de las dos pruebas puede reemplazar a la otra. Para concluir señalar que considerando nuestros resultados, pensamos que ambas exploraciones son complementarias, con una sensibilidad subóptima de la tiroglobulina en la detección de la afectación ganglionar loco-regional y del tejido tiroideo residual y un valor predictivo negativo que no permite realizar el seguimiento de pacientes con carcinoma diferenciado de tiroides, basándose únicamente en los resultados de este marcador tumoral.

\section{Bibliografía}

1. Aiello DP, Manni A. Thyroglobulin measurement vs iodine 131 totalbody scan for follow-up of well-differentiated thyroid cancer. Arch Intern Med 1990; 150: 437-9.

2. Ashcraft MW, Van Herle AJ. The comparative value of serum thyroglobulin measurements and iodine 131 total scan in the follow-up study of patients with treated differentiated thyroid cancer. Am J Med 1981; 71 : 807-14.

3. Black EG, Sheppard MC, Hoffenberg R. Serial serum thyroglobulin measurement in the management of differentiated thyroid carcinoma. Clin Endocrinol 1987; 13: 107-10.
4. Bolk JH, Bussemaker JK, Nieuwenhuizen Kruseman AC, De Vijlder JJM, Goslings BM. Thyroglobulin measurements in the follow-up of patients with differentiated thyroid carcinoma: comparison with quantitative radioactive iodine uptake and total body scans. Neth J Med 1985; 28: 340-6.

5. Botsch H, Glatz J, Schulz E, Wenzel KW. Long- term follow-up using serial serum thyroglobulin determinations in patients with differentiated thyroid carcinoma. Cancer 1983; 52: 1856-9.

6. Brendel AJ, Lambert B, Guyot M, Jeandot R, Dubourg H, Roger P, Wynchauks et al. Low levels of serum thyroglobulin after withdrawal of 
thyroid suppression therapy in the follow up of differentiated thyroid carcinoma. Eur J Nucl Med 1990; 16: 35-8.

7. Charles MA, Dodson LE Jr, Waldeck N, Hofeldt F, Ghaed N, Telepak $\mathrm{R}$ et al. Serum thyroglobulin levels predict total body iodine scan findings in patients with treated well-differentiated thyroid carcinoma. Am J Med 1980; 69: 401-7.

8. Dietlein M, Scheidhauer K, Voth E, Theissen P, Schicha H. Fluorine-18 fluorodeoxyglucose positron emission tomography and iodine-131 whole-body scintigraphy in the follow-up of differentiated thyroid cancer. Eur J Nucl Med 1997; 24: 1342-8.

9. Dietlein M, Scheidhauer K, Voth E, Theissen P, Schicha H. Follow-up of differentiated thyroid cancer; what is the value of FDG and sestamibi in the diagnostic algorithm? Nuklearmedizin 1998; 37: 12-7.

10. Fletcher, R. A. H.Clinical Epidemiology, $2^{\mathrm{a}}$ ed. Williams and Wilkins, Baltimore, 1988.

11. Franceschi M, Kusic Z, Franceschi D, Lukinac L, Roncevic S. Thyroglobulin determination, neck ultrasonography and iodine-131 wholebody scintigraphy in differentiated thyroid carcinoma. J Nucl Med 1996; 37: 446-51.

12. Galen RS, Gambino SR. Beyond normality: the predictive value and efficiency of medical diagnosis. New York: John Wiley and Sons. Inc, 1975.

13. Girelli ME, Busnardo B, Amerio R, Casara D, Betterle C, Piccolo M. Critical evaluation of serum thyroglobulin $(\mathrm{Tg})$ levels during thyroid hormone suppression therapy versus Tg levels after hormone withdrawal and total body scan: Results in 291 patients with thyroid cancer. Eur J Nucl Med 1986; 11: 333-5.

14. Girelli ME, Busnardo B, Amerio R, Scotton G, Casara D, Betterle C et al. Serum thyroglobulin levels in patients with well-differentiated thyroid cancer during suppression therapy: study on 429 patients. Eur J Nucl Med 1985; 10: 252-4.

15. Grant S, Luttrell B, Reeve T, Wiseman J, Wilmshurst E, Stiel J et al Thyroglobulin may be undetectable in the serum of patients with metastatic disease secondary to differentiated thyroid carcinoma. Follow-up of differentiated thyroid carcinoma. Cancer 1984; 54; 1625-8.

16. Lubin E, Mechlis-Frish S, Zatz S, Shimoni A, Segal K, Avraham A et al. Serum thyroglobulin and iodine-131 whole-body scan in the diagnosis and assessment of treatment for metastatic differentiated thyroid carcinoma. J Nucl Med 1994; 35: 257-62.

17. Mazzaferri EL. Editorial: treating high thyroglobulin with radioiodine: a magic bullet or a shot in the dark? J Clin Endocrinol Metab 1995; 80: 1485-7.

18. Mertens I J, De Klerk J M, Zelissen P M, Thijssen J H, Sie-Go D.M, Han $\mathrm{SH}$ et al. Undetectable serum thyroglobulin in a patient with metastatic follicular thyroid cancer. Clin Nucl Med 1999; 24: 346-9.

19. Moser E, Fritsch S, Braun S. Thyroglobulin and I-131 uptake of remaining tissue in patients with differentiated carcinoma after thyroidectomy. Nucl Med Commun 1988; 9: 262- 6.
20. Ozata M, Suzuki S, Miyamoto T, Tsuan Liu R, Fierro-Renoy F, Degroot LJ. Serum thyroglobulin in the follow-up of patients with treated differentiated thyroid cancer. J Clin Endocrinol Metab 1994; 79: 98-105.

21. Pacini F, Cetani F, Miccoli P, Mancusi F, Ceccarelli C, Lippi F et al Outcome of 309 patients with metastatic differentiated thyroid carcinoma treated with radioiodine. World J Surg 1994; 18: 600-4.

22. Ramanna L, Waxman AD, Brachman MB, Sensel N, Tanasescu DE Berman DS et al. Correlation of thyroglobulin measurements and radioiodine scans in the follow-up of patients with differentiated thyroid cancer. Cancer 1985; 55: 1525-9.

23. Roelants V, De Nayer P, Bouckaert A, Beckers C. The predictive value of serum thyroglobulin in the follow-up of differentiated thyroid cancer. Eur J Nucl Med 1997; 24: 722-7.

24. Ronga G, Fiorentino A, Paserio E, Signore A, Todino V, Tummarello MA et al. Can Iodine-131 whole-body scan be replaced by thyroglobulin measurement in the post-surgical follow-up of differentiated thyroid carcinoma? J Nucl Med 1990; 31: 1766-71.

25. Salvatori M, Saletnich I, Rufini V, Troncone L. Unusual false-positive radioiodine whole-body scans in patients with differentiated thyroid carcinoma. Clin Nucl Med 1997; 22: 380-4.

26. Scheneider AB, Line BR, Goldman JM, Robbins J. Sequential serum thyroglobulin determinations, 131 I scans, and 131 I uptakes after triiodothyronine withdrawal in patients with thyroid cancer. J Clin Endocrinol Metab 1981; 53: 1199- 206.

27. Schlumberger M, Challeton C, De Vathaire F, Travagli JP, Gardet P, Lumbroso JD et al. Radioactive iodine treatment and external radiotherapy for lung and bone metastases from thyroid carcinoma. J Nucl Med 1996; 37: 598-605.

28. Schlumberger M, Tubiana M, De Vathaire F, Hill C, Gardet P, Travagli JP et al. Long-term results of treatment of 283 patients with lung and bone metastases from differentiated thyroid carcinoma. J Clin Endocrinol Metab 1986; 63: 960-7.

29. Spencer C, Wang C. Thyroglobulin measurement. Techniques, clinical benefits and pitfalls. Endocrinol Metab Clin North Am 1995; 24: 841.

30. Sutter CW, Masilungan BG, Stadalnik RC. False-positive results of I131 whole-body scans in patients with thyroid cancer. Semin Nucl Med 1995; 25: 279.

31. Thomas-Morvan C, Carayon P, Schlumberger M, Vignal A, Tubiana M Thyrotrophin stimulation of adenylate cyclase and iodine uptake in human differentiated thyroid cancer. Acta Endocrinol 1982; 101: 25-31.

32. Van-Herle AJ, Agatep ML, Padua DN 3d, Totanes TL, Canlapan DV, Van Herle HM et al. Effects of 13 cis-retinoic acid on growth and differentiation of human follicular carcinoma cells. (UCLA RO $82 \mathrm{~W}-1$ ) in vitro. J Clin Endocrinol Metab 1990; 71: 755-63.

33. Van Herle AJ, Van Herle IS, Greipel MA. An international cooperative study evaluating serum thyroglobulin standards. J Clin Endocrinol Metab 1985; 60: 338-43 